\title{
ONREL \\ Design of a Microgrid Transition Controller II: System Recovery Under Abnormal Conditions
}

Jing Wang, Annabelle Pratt, and Murali Baggu

Power Systems Engineering Center, National Renewable Energy Laboratory Golden, CO 80401, USA

\section{Abstract}

This paper presents a microgrid transition controller for managing emergency operation when the microgrid experiences a system blackout caused by an internal or external fault. The developed transition controller consists of various application function blocks (AFBs), including normal operation, emergency operation, and coordination between them. The developed microgrid transition controller is validated by a sample microgrid, and two test cases are investigated: islanded black start and grid-connected black start. The simulation results demonstrate the feasibility and effectiveness of the proposed controller for handling emergency operation and transitioning to normal operation.

\section{Overview of Microgrid Operation Under} Different Conditions

In normal conditions, the microgrid system operates in any mode inside the inner loop. The outer loop is the emergency operation mode, which is caused by various reasons, such as internal/external faults, unscheduled outages, or voltage/frequency transients.

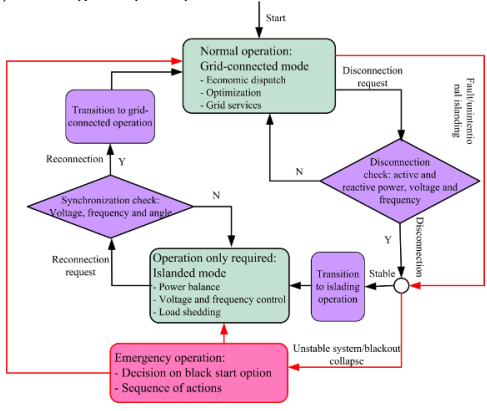

AFB\#1: Operation Mode Management

Distribution network operator (DNO) signal processing, point of common coupling (PCC) disconnection enable signal, and PCC reconnection enable signal and operation mode decision

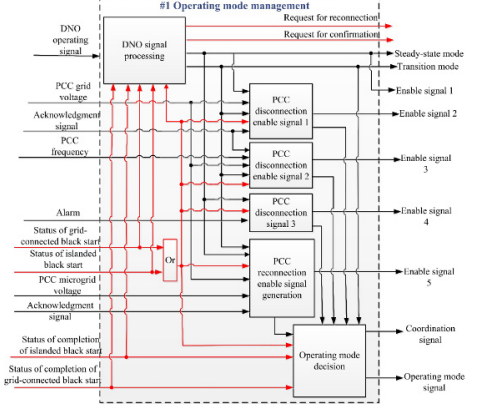

Design of the Microgrid Transition

\section{Controller}

- Layered structure: DNO control room, supervisory level, device control level, field level

- AFBs and coordination among them.

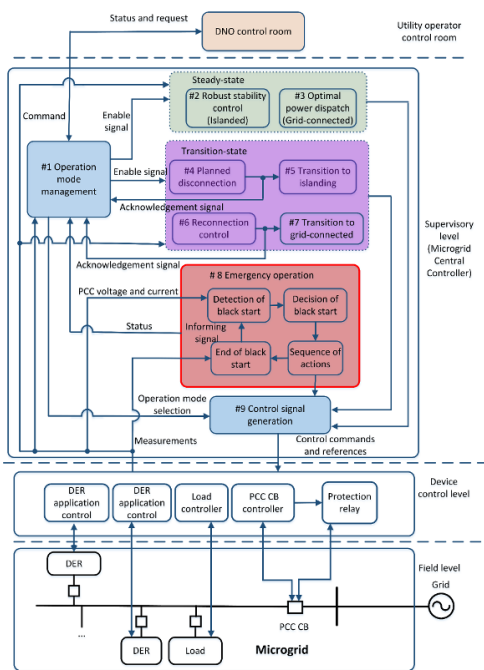

Schematic diagram of the transition controller

\section{AFB\#8: Emergency Operation}

Detection of blackout

- Decision of black-start option

- Sequences of black start: grid-connected or islanded

- Check completion of black start

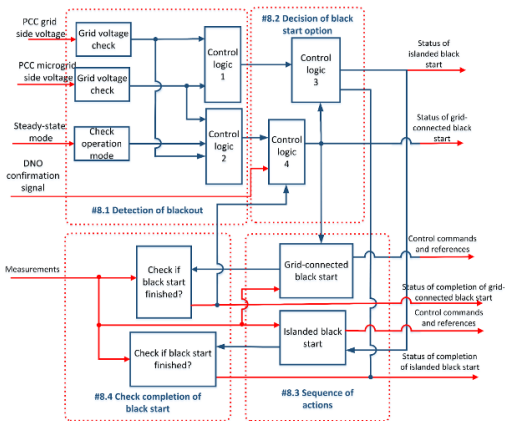

\section{Results}

Scenario 1: Islanded Black Start

The microgrid system starts from operating in grid-connected mode. Then an external fault is triggered and removed after a certain amount of time. The microgrid controller detects the situation, opens the PCC circuit breaker, and black starts the system in islanded mode.

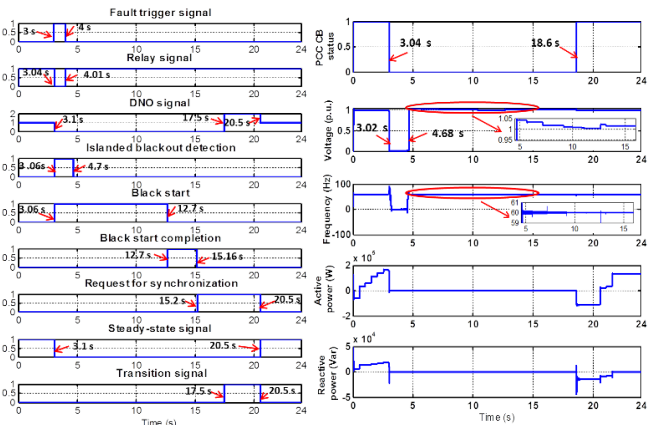
Coordination signals inside the Measurements at PCC: circuit microgrid transition controller

heasurements at PCC: circut Scenario 2: Grid-Connected Black Start

The microgrid system starts from operating in islanded mode. Then an internal fault is triggered and removed after a certain amount of time. The microgrid controller detects the situation, turns off all generation, closes the PCC circuit breaker, and black starts the system in grid-connected mode.

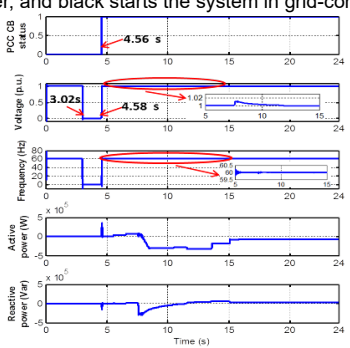

Measurements at PCC: circuit breaker status, voltage, etc.

\section{Conclusions}

The developed microgrid transition controller focuses on operation functionalities for emergency operation, which is validated by numerical simulation in MATLAB/Simulink. The results show that the developed microgrid transition controller can handle emergency operation and transition the system to normal operation.

Additional Reading Jing Wang, Changhong Zhao, Annabelle Pratt, and Murali Baggu, 2018. "Design of an Advanced Energy
Management System for Microgrid Control Using a State Machine." Applied Energy 228: $2407-2421$. 